\title{
Motivaciones para la práctica deportiva en escolares federados y no federados Motivations for practicing sports in federate and non-federate students
}

\author{
Manuel Isorna Folgar*, Antonio Rial Boubeta** y Raquel Vaquero-Cristóbal ${ }^{* * *}$ \\ *Universidad de Vigo. **Universidad de Santiago de Compostela. ***Universidad Católica San Antonio de Murcia
}

Resumen: En este artículo se analiza el perfil motivacional hacia la práctica de ejercicio físico entre los escolares de varios centros educativos a través de la Teoría de la Autodeterminación de Deci y Ryan, diferenciando los resultados en función del sexo, el modo de la práctica deportiva (federado vs. no federado) y el deporte practicado; así como conocer la influencia de la implantación de una unidad de iniciación en el ámbito escolar en la práctica de este deporte en horario extracurricular. Un total de 306 alumnos/as de $5^{\circ}$ de primaria a $4^{\circ}$ de la Educación Secundaria Obligatoria (ESO) con edades comprendidas entre los 9 y 16 años, los cuales pertenecían a centros educativos en los cuales se había dado una unidad didáctica de iniciación al piragüismo, completaron la versión en castellano del BREQ-2. Los resultados mostraron que sólo el 57.2\% de los sujetos practicaban deporte federado, siendo la mayoría de ellos hombres. Se ha encontrado que los estudiantes tenían puntuaciones altas en motivación intrínseca, moderadas en regulación identificada y regulación introyectada, bajas en regulación externa y muy bajas en desmotivación, mostrando quienes practican deporte federado unos niveles más altos de motivación intrínseca y la regulación identificada que los que hacían deporte no federado. Los hombres mostraron una mayor motivación intrínseca que las mujeres. No se encontraron diferencias en función de la modalidad deportiva practicada. El fútbol fue el deporte más practicado, seguido del piragüismo y el baloncesto.

Palabras clave: deporte, deporte federado, adolescentes, autodeterminación, ejercicio físico, piragüismo.

Abstract: This paper analyzes the motivational profile in physical exercise among students from various schools with the Deci \& Ryan' selfdetermination theory. The results were divided according to sex, mode of sport (federated vs non-federated) and the sport modality, as well as know the influence of the implantation of an initiation unit in the school in the practice of this sport in extracurricular schedule. A total of 306 male / female students from Elementary $5^{\text {th }}$ to $4^{\circ} \mathrm{ESO}$, with ages between 9 and 16, who belonged to schools in which there had been teaching a didactical unit of initiation to canoeing, completed the Spanish version of the BREQ-2. The results showed only a 57.2\% of the students did federate sport, most of them being men. Students had high scores in intrinsic motivation, moderate scores in identified regulation and introjected regulation, low scores in external regulation, and very low scores in demotivation. Federated sport practitioners showed higher levels of intrinsic motivation and identified regulation than those who did not do federated sport. Men showed greater intrinsic motivation than women. No differences were found depending on the sport modality practiced. Fooball is the most popular sport, followed by canoeing and basketball.

Key words: sport, federated sport, teenagers, self-determination, physical exercise, canoeing.

\section{Introducción}

Diversos estudios revelan los beneficios que proporciona la práctica físico-deportiva moderada en el ámbito físico, fisiológico, psicológico y social (American College of Sports Medicine, 2000; Fox, 2000). Seha demostrado que con la práctica físico-deportiva aumenta la autoestima, creando un efecto positivo en el bienestar del sujeto practicante; por el contrario, el sedentarismo ha sido asociado con estados depresivos y problemas de salud (Sonstroem \& Potts, 1996; Varo, Martínez \& Martínez-González, 2003). Sin embargo, y a pesar de estos beneficios, el sedentarismo se está configurando como un importante problema en la infancia y en la adolescencia, predominado los sedentarios frente a los físicamente activos (García-Ferrando, 2006b).

En muchos casos se da por supuesto que los niños ya realizan ejercicio físico en las clases de educación física, y que por lo tanto, ya podríamos hablar de una niñez y primera adolescencia activa. Sin embargo, esta suposición no está apoyada por la investigación empírica. Los datos procedentes de numerosos estudios informan que el ejercicio físico realizado en el colegio, por sí sólo, no es suficiente para promover beneficios saludables óptimos. Esto es debido a que los niños durante sus clases de educación física no dedican el tiempo suficiente a practicar ejercicio físico (Castillo, Balaguer \& Tomás, 1997). Parcel, Simons, O’Hara, Baranowski, Kolbe y Bee (1987) encontraron que en una clase de 30 minutos de educación física, sólo se dedicaban 2 minutos a una actividad física vigorosa. Por lo tanto, las clases de educación física son necesarias aunque no suficientes para mantener a los niños/adolescentes saludables (física, psicológica y socialmente); por lo que se debe complementar la actividad realizada en el colegio con más ejercicio físico

Fecha recepción: 13-06-13- Fecha envío revisores: 15-06-13- Fecha de aceptación: 28-07-13 Manuel Isorna Folgar

Lagoas, $\mathrm{s} / \mathrm{n}$.

Lagoas, s/n.

isorna.catoira@uvigo.es fuera ámbito escolar. Esto ha llevado a algunos investigadores a diferenciar entre práctica de actividades físicas dentro y fuera del colegio (Wold, 1995).

El problema de la inactividad se agrava con la edad, pues numerosas investigaciones muestran que a medida que avanza la adolescencia, se produce un deterioro del estilo de vida saludable. Se observa que los adolescentes de 11 y 13 años presentan estilos de vida más saludables (no beben, no fuman, hacen deporte) que los adolescentes de 15 y 17 años (Macías \& Moya, 2002). En algunas investigaciones se ha cifrado la disminución del ejercicio físico con la edad, en casi un $50 \%$ entre los 6 y los 16 años (García-Ferrando, 2006b). Por tanto, son la adolescencia y el inicio de la edad adulta las etapas de abandono deportivo más frecuentes (Balaguer, Pastor \& Moreno, 1999). La tendencia es clara en ambos sexos, pero particularmente en las chicas, quienes presentan hábitos más sedentarios (Macías \& Moya, 2002) y además hacen ejercicio físico con una menor frecuencia (Balaguer, et al., 1999).

Además, en los últimos años la reducción de la práctica de ejercicio físico de manera habitual se ha visto reforzada como consecuencia de la cantidad de horas que los niños pasan sentados en la escuela, el uso del transporte motorizado para desplazarse y la proliferación de actividades de ocio tecnológico que incitan al sedentarismo. Por tanto, es vital tener en cuenta las conductas sedentarias como parte de la estrategia en la prevención del desarrollo prematuro de riesgo cardiovascular en la infancia y la adolescencia, como un factor independiente del ejercicio físico realizado, y resulta imprescindible realizar recomendaciones con el fin de reducir el tiempo que niños y adolescentes permanecen inactivos. De hecho, sólo el $28 \%$ de los niños y el $16 \%$ de las niñas españolas entre 12 y 17 años realizan la cantidad de ejercicio físico recomendado para su edad, es decir, 60 minutos de ejercicio al día, al menos cinco días a la semana (Martínez, Joey, Gómez, Veses, Marcos \& Veiga, 2010).

Numerosas investigaciones asignan una importancia vital a las motivaciones en la participación de los niños, adolescentes y jóvenes en la práctica físico deportiva en el tiempo de ocio. Los trabajos que han 
estudiado los predictores para que el sujeto sea activo han estructurado el análisis entre factores personales, sociales y ambientales (Sallis, et al. 1992). En relación a los factores personales se ha encontrado que son la percepción de la competencia física o deportiva, la salud percibida y la forma física percibida, los que predicen en mayor medida la práctica de ejercicio físico y deporte (Moreno, Cervelló \& González-Cutre, 2007). Otro de los motivos con gran importancia, es el gusto por practicar una determinada modalidad deportiva y el conseguir el dominio de ésta (Armenta, Prieto \& Morilla, 1993).

El proceso de socialización es otro de los procesos básicos que interviene en la adquisición de un modelo de vida activo. Aquí los padres y otras personas representativas para el sujeto, como sus hermanos y hermanas, los amigos y los educadores, juegan un papel fundamental (Nuviala, Ruíz \& García, 2003; Sallis, Prochaska \& Taylor, 2000; Van Der Horst, Paw, Twisk \& Van Mechelen, 2007). Varias investigaciones han concluido que los niños y los adolescentes son más propensos a ser físicamente activos cuando sus familiares y/o amigos participan en actividades físico-deportivas de forma regular (Piéron 2002; Van Der Horst, et al., 2007; Wuerth, Lee \& Alfermann, 2004), les impulsan a practicar (Biddle \& Goudas, 1996) o incluso cuando practican deporte con ellos (Shoopshire \& Carroll, 1997). Otros factores sociales que se consideran predictores importantes para la práctica deportiva son la diversión y la mejora de las relaciones sociales, especialmente de las amistades (Pallarés, 1998; Trigo, 1990). En relación con este último punto, la facilidad para hacer amigos, tanto dentro como fuera de la escuela y la frecuencia semanal de estar con los amigos después del colegio, han aparecido asociadas con la práctica deportiva del adolescente (Wold., 1995). También se ha encontrado que el hecho de llevar a un niño al club deportivo, de hacerlo socio y apoyarlo son factores que propiciarán la práctica de actividades físico-deportivas (Romero, Garrido \& Zagalaz, 2009)

En cuanto a los factores ambientales, el acceso a instalaciones deportivas ha sido correlacionado positivamente con la práctica de ejercicio físico de los jóvenes de todas las edades (García-Ferrando, 2006b; Zakarian, Hovell, Hofstetter, Sallis \& Keating, 1994). Castilloy Balaguer (1998) encontraron que ser miembro de algún club deportivo está fuertemente asociado con altos niveles de práctica de ejercicio físico en niños y adolescentes, sobre todo en el caso de los chicos, quienes practican más deporte federado que las chicas (Pavón \& Moreno, 2008). El interés de los varones por la competición y el reclamo a la misma que les hace la sociedad pueden ser los motivos por los cuales los chicos están más federados que las chicas (García-Ferrando, 2006a).

La relación entre estos tres grupos de factores es compleja, interaccionando unos con otros de forma diferenteen cada sujeto (Brustad, 1996; Roberts, 1992), derivando la elección de una actividad deportiva u otra, la intensidad de la práctica de dicha actividad, la persistencia en la tarea, el rendimiento y la motivación de cómo se relacionen estos tres factores (Balaguer, 1994).

La mayoría de los estudios relacionados con la motivación se han apoyado en la teoría de la Autodeterminación (Balaguer, Castillo, \& Duda, 2008; Moreno, Cano, González-Cutre, Cervelló \& Ruiz, 2009; Ryan \& Deci, 2000) y de las metas de logro (Nicholls, 1989), mostrando importantes diferencias motivacionales en los individuos a la hora de participar en actividades físico-deportivas, repercutiendo considerablemente dicha orientación motivacional no sólo en el inicio, sino más aún en la continuidad y el abandono de las mismas (Ryan \& Deci, 2000). En base a estas teorías se puede hablar de sujetos intrínsecamente motivados (aquellos que participan por la satisfacción que les genera dicha actividad deportiva), sujetos motivados extrínsecamente (cuando su compromiso en la actividad se fundamenta en razones externas: presión padres, compañeros, entrenadores, etc.) y sujetos desmotivados (caracterizados por falta de interés hacia la práctica y por sentimientos de frustración). Asu vez, Ryan y Deci (2000) recogen diferentes subtipos de motivación extrínseca. Así, el sujeto puede considerar que la actividad es importante pero no placentera (regulación identificada), puede practicar por sentimientos de culpabilidad (regulación introyectada) o, simplemente, por presiones externas (regulación externa).
Parece ser que los jóvenes deportistas se acercan más al deporte por motivos intrínsecos que extrínsecos (Villamarin, Mauri \& Sanz, 1998). Por otro lado, es la falta o pérdida de motivación uno de los motivos que mayor peso tiene en la literatura, junto con la falta de tiempo y las lesiones (o problemas de salud) en referencia a por qué los adolescentes se pasan del «deporte federado» al «no federado» o lo abandonan por completo (Cantallops, Ponseti, Vidal, Antoni \& Palou, 2012). Teniendo en cuenta que en el caso del deporte federado existe una mayor continuidad a lo largo de la temporada, los días de entrenamiento y competición son superiores a los de la práctica no federada, y la intensidad de las sesiones es más elevada, la promoción del deporte federado entre los adolescentes puede ser un buen medio para evitar el sedentarismo. Sin embargo, es necesario capacitar y formar a todos aquellos que tienen alguna responsabilidad directa con la población diana de tal forma que tengan la capacidad para motivar a las personas y ayudarlas a descubrir y dirigir adecuadamente su motivación. En esteámbito, los profesionales que trabajan con los niños y adolescentes deben tener una comprensión profunda de los factores motivacionales que dirigen la conducta y de los procedimientos y estrategias necesarios para crear un clima adecuado y positivo para aumentar la efectividad en el propio individuo y en los grupos (Cantallops, et al., 2012).

Bajo estas premisas y considerando la aportación realizada por Sansone y Harackiewicz (2000) en relación a la necesidad de estudiar los perfiles motivacionales de los sujetos bajo similares condiciones ambientales, así como la necesidad de satisfacer los nuevos valores deportivos de la sociedad, los objetivos de la presente investigación fueron los siguientes: 1. Analizar la distribución de niños/adolescentes que practican ejercicio físico/deporte en función de si están federados, de su sexo y de la modalidad deportiva que practicaban; 2 . Comparar los niveles de autodeterminación en función de la práctica de deporte federado o no federado y/o del sexo; 3. Realizar una comparativa según las variables «a priori» antes descritas sobre los niveles de autodeterminación. 4. Comprobar si existe un perfil motivacional diferente según el deporte practicado. 5. Analizar la influencia de la implantación de una unidad didáctica de piragüismo en la práctica de este deporte en el periodo extraescolar.

\section{Método}

\section{Participantes}

Doscientos ochenta y siete escolares (147 hombres y 140 mujeres) de seis centros educativos de las provincias de Pontevedra y A Coruña, con edades comprendidas entre los 9 y los 16 años (media de edad: $12.60 \pm 1.83$ años), participaron en el estudio. Los criterios de inclusión fueron realizar algún tipo de deporte o ejercicio físico a nivel extraescolar.

\section{Instrumentos}

La recogida de datos se realizó mediante un cuestionario anónimo que se autocumplimentaba en la hora de educación física en la propia aula, el cual tenía una duración aproximada de una hora. Durante la administración del cuestionario el profesor/a de educación física estaba presente. Se seleccionan estos C.P.I. «ad hoc» porque presentaban como característica común la iniciación al piragüismo en su diseño curricular, ya que en todos estos centros se aplicaron unidades didácticas de iniciación al piragüismo, además de otros deportes.

Previamente a las mediciones, todos los participantes fueron informados sobre los procedimientos y sus tutores firmaron, voluntariamente, un consentimiento informado. Además, se les preguntó el sexo, si practican ejercicio físico/deporte de manera federada o no federada y que tipo de modalidad.

El instrumento utilizado para conocer los niveles de autorregulación fue el Cuestionario de Regulación de la Conducta en el Ejercicio Físico2 (BREQ-2), versión traducida al español y validada(Moreno, Cervelló \& Martínez, 2007) del instrumento Behavioral Regulation in Exercise Questionnaire-2 (BREQ-2) (Markland \& Tobin, 2004). Dicho cuestionario estaba compuesto por un total de 19 ítems, agrupados en cinco factores que representan diferentes tipos de motivación: regulación intrínseca, regulación identificada, regulación introyectiva, regulación 
externa y desmotivación. Todos los ítems se respondieron a través de una escala tipo Likert cuyo rango va desde el valor 0 (nada verdadero) a 4 (totalmente verdadero).

\section{Análisis estadístico}

La distribución de los datos fue inicialmente valorada mediante el test de normalidad de Kolmogorov-Smirnov. Puesto que las variables seguían una distribución normal, se realizó un análisis estadístico en base a pruebas paramétricas. Para la obtención de los resultados se realizó una estadística descriptiva con la cual se hallaron los valores medios y desviación estándar(SD). Para establecer las diferencias en las variables en función que realizaran deporte federado o no federado o del sexo se realizó una prueba $t$ de student para muestras independientes. Para analizar las diferencias en función de ambos factores se realizó una pruebaANOVAde dos factores. Para hallar las diferencias en función de la práctica deportiva realizada la prueba elegida fue el ANOVA de un factor. Se estableció un valor de $p<.05$ para determinar la significación estadística. El análisis estadístico fue realizado mediante el paquete estadístico SPSS (v. 15.0; SPSS Inc., IL).

\section{Resultados}

De los 287 sujetos, 165 (57.5\%) practicaban algún deporte federado y 122 sujetos (42.5\%) practicaban deporte o ejercicio físico en actividades organizadas por las Asociaciones de padres y madres de alumnos y escuelas deportivas municipales, por su cuenta o en compañía de sus padres y/o amigos. Al analizar los datos en función del sexo se encontró que un $72 \%$ de los varones estaban federados, mientras que un 41.1\% de las mujeres tenían ficha federativa, siendo las diferencias observadas en el número de federados en función del sexo estadísticamente significativa $(p<.001)$. No se encontraron diferencias en el número de federados al analizar la muestra por edades ( $p=.62$ ), siendo el promedio de edad de los que practican deporte federado (12.65 \pm 3.65 años) muy similar los que no lo practican (12.54 \pm 3.60 años).

En la figura 1 se muestran los promedios obtenidos por los encuestados en las diferentes subescalas. Se encontró que los participantes tenían puntuaciones altas en motivación intrínseca, moderadas en regulación identificada y regulación introyectada, bajas en regulación externa y muy bajas en desmotivación; con lo que se puede afirmar que los deportistas presenta un «perfil autodeterminado» (Moreno, Cervelló \& González-Cutre, 2007).

Al analizar estos datos en función de la práctica de deporte federa-

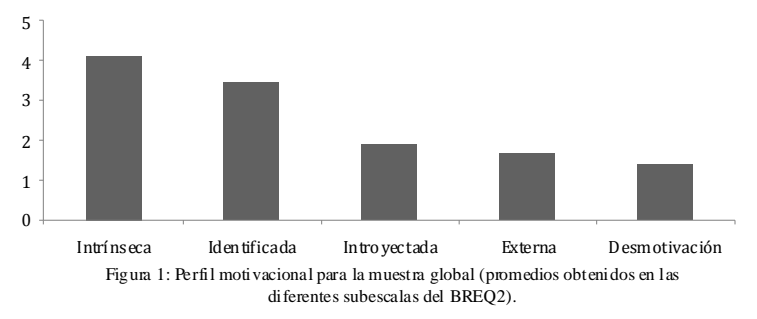

Tabla 1: Nivel de Autodeterminación según el tipo de deporte practicado: federado o no federado.

\begin{tabular}{|c|c|c|c|c|}
\hline & $\begin{array}{l}\text { DEPORTE FEDERADO } \\
\text { (Media } \pm \text { SD) }\end{array}$ & $\begin{array}{l}\text { DEPORTE NO FEDERADO } \\
\text { (Media } \pm \text { SD) }\end{array}$ & $\mathrm{t}$ & Sig \\
\hline Intrínseca & $4.34 \pm .70$ & $3.84 \pm .95$ & 4.94 & .001 \\
\hline Identificada & $3.55 \pm .72$ & $3.29 \pm .83$ & 2.80 & .005 \\
\hline Introyectada & $1.85 \pm .97$ & $1.94 \pm .94$ & -0.712 & .477 \\
\hline Externa & $1.59 \pm .81$ & $1.71 \pm .79$ & -1.27 & .203 \\
\hline Desmotivación & $1.39 \pm .61$ & $1.45 \pm .63$ & .885 & .377 \\
\hline & HOMBRE (Media \pm SD) & \multicolumn{2}{|c|}{ Tabla 2: Medias y desviaciones de Autodetermin ación según el sexo. } & Sig \\
\hline Intrínseca & $4.27 \pm 0.77$ & $3.98 \pm 0.91$ & 2.95 & 0.005 \\
\hline Identificada & $3.48 \pm 0.81$ & $3.39 \pm 0.74$ & 0.999 & 0.319 \\
\hline Introyectada & $1.94 \pm 1.03$ & $1.84 \pm 0.87$ & 0.862 & 0.389 \\
\hline Externa & $1.69 \pm 0.89$ & $1.59 \pm 0.71$ & 1.01 & 0.312 \\
\hline Desmotivación & $1.46 \pm 0.66$ & $1.37 \pm 0.57$ & 1.17 & 0.241 \\
\hline
\end{tabular}

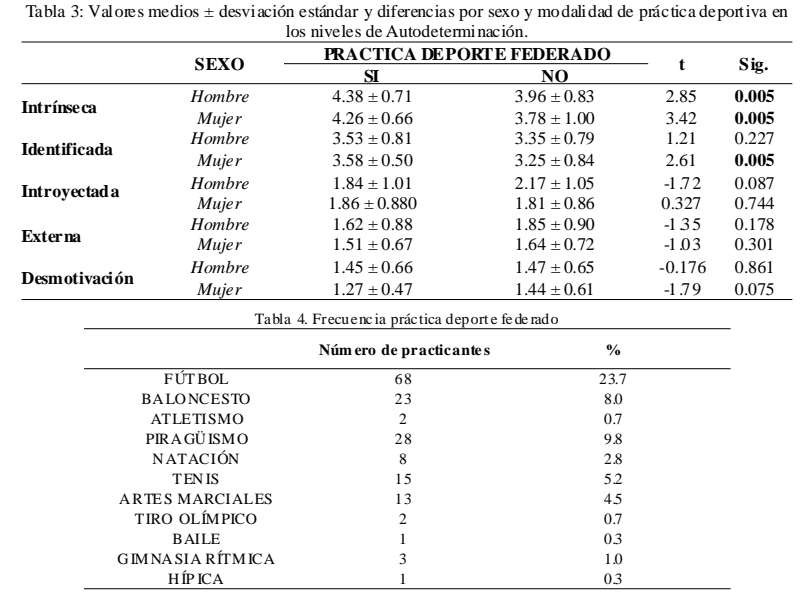

do o no federado se encontraron diferencias significativas en la motivación intrínseca $(p<.001)$ y la regulación identificada $(p<.05)$, siendo los valores de deportistas federados más altos en ambas variables. En el resto de niveles de autodeterminación no se hallaron diferencias significativas (tabla 1).

Al dividir los datos por sexo, se obtuvo que sólo hay diferencias significativas en la motivación intrínseca $(p<.01)$, siendo los hombres los que puntuaron más alto (tabla 2).

Al combinar las variables sexo y práctica de deporte federado o no, se comprobó que los chicos y chicas que practican deporte federado tienen puntuación más altas en los niveles de motivación intrínseca $(p<.01)$ en relación al grupo del mismo sexo pero que no realizaba deporte federado; mientras que las mujeres que hacen deporte federado tienen puntuación significativamente más altas en la autodeterminación identificada $(p<.01)$ que las que hacen deporte no federado. En el resto de niveles no se encontraron diferencias significativas (tabla 3).

La distribución de los deportistas federados en función de la modalidad deportiva practicada se encuentra en la tabla 4. El fútbol fue el deporte federado con mayor número de practicante, seguido del piragüismo y baloncesto.

Con el ánimo de explorar la existencia de posibles diferencia entre deportes, se realizó una comparativa entre los cinco deportes que contaban con mayor número de practicantes en la muestra disponible (fútbol, baloncesto, piragüismo, tenis y artes marciales) de las variables del plano motivacional (tabla 5). No se encontraron diferencias signifi-

\begin{tabular}{|c|c|c|c|c|}
\hline & DEPORTE & Media \pm SD & $\mathbf{F}$ & Sig. \\
\hline & Fútbol & $4.40 \pm 0.60$ & & \\
\hline & Baloncesto & $4.51 \pm 0.55$ & & \\
\hline \multirow[t]{5}{*}{ Intrínseca } & Piragüismo & $4.21 \pm 0.84$ & 0.847 & 0.497 \\
\hline & $\begin{array}{l}\text { Tenis } \\
\text { Th }\end{array}$ & $4.43 \pm 0.51$ & & \\
\hline & Artes Marciales & $4.51 \pm 0.75$ & & \\
\hline & Fútbol & $3.43 \pm 0.77$ & & \\
\hline & Bal oncesto & $3.85 \pm 0.59$ & & \\
\hline \multirow[t]{5}{*}{ Identificada } & Piragüismo & $3.51 \pm 0.76$ & 1.60 & 0.175 \\
\hline & Tenis & $3.60 \pm 0.52$ & & \\
\hline & Artes Marciales & $3.67 \pm 0.68$ & & \\
\hline & Fútbol & $1.90 \pm 0.99$ & & \\
\hline & Bal oncesto & $1.84 \pm 1.11$ & & \\
\hline \multirow[t]{5}{*}{ Introyectada } & Piragüismo & $1.92 \pm 0.98$ & 0237 & 0.917 \\
\hline & Tenis & $1.66 \pm 0.78$ & & \\
\hline & Artes Marciales & $1.76 \pm 0.91$ & & \\
\hline & Fútbol & $1.57 \pm 0.87$ & & \\
\hline & Bal oncesto & $1.43 \pm 0.70$ & & \\
\hline \multirow[t]{5}{*}{ Externa } & Piragüismo & $1.83 \pm 1.02$ & 1.27 & 0.284 \\
\hline & Tenis & $1.50 \pm 0.50$ & & \\
\hline & Artes Marciales & $1.28 \pm 0.36$ & & \\
\hline & Fútbol & $1.43 \pm 0.65$ & & \\
\hline & Bal oncesto & $1.35 \pm 0.56$ & & \\
\hline \multirow[t]{3}{*}{ Desmotivación } & Piragüismo & $1.36 \pm 0.61$ & 1.15 & 0.334 \\
\hline & & $1.08 \pm 0.18$ & & \\
\hline & Artes Marciales & $1.30 \pm 0.37$ & & \\
\hline
\end{tabular}

cativas entre deportes en ninguno de los ítems.

\section{Discusión}

El principal hallazgo del presente estudio fue que los sujetos que realizaban deporte federado presentaban un perfil autoderminado, el cual se caracterizaba por que tenían altas puntuaciones en la motivación intrínseca y la regulación identificada y puntuaciones más bajas en la regulación introyectada, externa y la desmotivación que los que practicaban deporte no federado. Estudios previos han demostrado que los 
deportistas con un perfil autodeterminado perciben que las repercusiones personales que la práctica deportiva les acarrea son muy positivas, presentando en general: mayor disfrute, esfuerzo, afecto positivo, actitud positiva hacia la participación deportiva, intenciones más fuertes y autodeterminadas para seguir practicando deporte durante mucho tiempo, una mayor satisfacción y son más persistentes que aquellos sujetos quepresentan un perfil no autodeterminado (Sarrazin, Vallerand, Guillet, Pelletier \& Cury, 2002; Vlachopoulos, Karageorghis \& Terry, 2000; Wilson, Rodgers, Fraser \& Murray, 2004). Así pues, los sujetos que practican deporte federado se divierten o consideran que el deporte es importante para su vida (salud, relaciones sociales) y en general muestran más interés, emociones positivas, estados psicológicos óptimos (flow), concentración, esfuerzo, rendimiento durante su práctica y ante todo, intención de seguir practicándolo.

Es de destacar la escasa participación de las mujeres en la práctica física deportiva, sobre todo de manera federada, lo cual corrobora los resultados encontrados en otros estudios (Castillo \& Balaguer, 1998; Navarro, Ojeda, Navarro, López, Brito \& Ruiz, 2012; Nuviala, et al., 2003; Pavón \& Moreno, 2008). Si a esto se añade que se produce una disminución de la práctica físico-deportiva con el paso de la edad, sobre todo en el paso de primaria a secundaria (Hernández, Ferrando, Quílez, Aragonés \& Terreros, 2010), se presenta un problema de difícil solución. Esta situación hace que sea necesaria una intervención educativa que permita a las niñas/adolescentes, padres y sociedad en general, concienciarse del valor y la importancia de ocupar de forma positiva y activa su tiempo libre. La escuela es el principal responsable, pero no el único, de encauzar y dar solución a la educación del tiempo libre, ya que la escuela y demás organizaciones y personas relacionadas con la educación y política deportiva deben responder a las aspiraciones y necesidades y retos sociales.

Estos datos pueden estar relacionados con los motivos que llevan a los jóvenes a practicar alguna actividad físico-deportiva. Los varones apuntan en mayor grado que las mujeres que los motivos de aprobación social, de afiliación y de demostración de sus capacidades son razones muy importantes a la hora de hacer deporte (Castillo, Balaguer \& Duda, 2000). Por tanto, los varones suelen mostrar mayor orientación disposicional al ego, estando esta variable motivacional relacionada con los motivos extrínsecos de la práctica deportiva (Castillo \& Balaguer, 2001). Esto ha sido confirmado en la presente investigación ya que los hombres presentan valores más altos de motivación extrínseca, aunque las diferencias respecto a las mujeres no son significativas.

Por otra parte, en muchas culturas occidentales, el deporte y el ejercicio físico son considerados como parte del ámbito masculino y ser atleta, a menudo, como un papel masculino más que femenino (McPherson, Curtis \& Loy, 1989). Como consecuencia de esto en los muchachos, el estímulo y la recompensa que los agentes socializantes ejercen promoviendo la práctica físico-deportiva es más fuerte que en las chicas (Greendorfer \& Lewko, 1978). Además, los distintos métodos de socialización de los niños les conducen a las actividades convenientes para el varón, es decir, aquellas que implican contacto, competición y fuerza corporal, mientras que para las mujeres se orientan hacia actividades convenientes para ellas, alegando estética e individualidad (Greendorfer \& Lewko, 1978). Esta influencia social se ve reflejada en la práctica deportiva. Raudsepp y Viira (2000) confirman que los varones participaron aún más en los deportes de equipo, como fútbol y baloncesto, mientras que las mujeres, sobre todo, practicaron natación, aeróbic y bicicleta. Estos hechos han condicionado totalmente las posibilidades de práctica de las mujeres, provocando actitudes y motivaciones muy peculiares, que están dando lugar en este momento a comportamientos específicos que, podríamos decir, responden a necesidades e intereses propios de la cultura masculina en los que la práctica física deportiva por parte de las mujeres no tiene lugar.

Otra posible causa/consecuencia de esta falta de adherencia de la mujer a la práctica deportiva es la enorme desigualdad en el tratamiento del deporte femenino en los medios de comunicación y más en un momento de avances sociales y normativos encaminados a garantizar la igualdad de hombres y mujeres en todos los ámbitos de la vida (García-
Ferrando, 2006a).

En la presente investigación los hombres también mostraron valores más altos de motivación intrínseca que las mujeres, habiendo diferencias significativas entre ambos grupos. Nuestros resultados son concordantes con los encontrados por Palou, Ponseti, Gili, Borras y Vidal (2005), quienes encontraron que los motivos y las actitudes hacia la práctica físico-deportiva varían en función del sexo, siendo más positivos y más favorables hacia la práctica los chicos, lo que les lleva además a presentar una implicación más autodeterminada que la población femenina.

Otro aspecto que cabe destacar es el alto porcentaje de niños y adolescentes que realizan deporte no federado, sobre todo entre las mujeres. Numerosos estudios han demostrado que entre la población española sólo un grupo reducido, un $4 \%$, señala como motivo para realizar deporte «el gusto por la competición», lo que indica que el afán competitivo es minoritario. El carácter unidimensional de la actividad competitiva victoria y/o rendimiento puede explicar, en parte, las decepciones mostradas por los adolescentes en cuanto no satisfacen sus expectativas, de ahí que otras actividades no competitivas e individualizadas, que eliminan los aspectos directamente relacionados con la búsqueda del perfeccionamiento y la victoria están alcanzando mayores cuotas de participación (Nuviala, et al., 2003). Por otra parte, las mujeres suelen señalar la estética, la mejora de la salud y de las relaciones sociales como los principales factores que les llevan a la realización de ejercicio físico/deporte (García-Ferrando, 2006a; Hellín, Moreno \& Rodríguez, 2004). Es necesario adaptar el deporte «tradicional» a estas nuevas demandas de las mujeres para conseguir su incorporación y mantenimiento en la práctica físico deportiva; dando de esta forma respuesta al cambio social que se está experimentando en los últimos tiempos en el ámbito deportivo, paralelo al cambio de mentalidad de la población joven, que busca con la actividad física la evasión y liberación de la vida cotidiana.

El fútbol es el deporte federado con mayor número de practicantes, seguido del piragüismo y baloncesto. Cabe destacar la alta posición del piragüismo en este trabajo, algo que contrasta con lo encontrado en investigaciones anteriores (García-Ferrando, 2006b). En este sentido, se puede afirmar que la instauración de unidades didácticas en los centros educativos es fundamental para la captación temprana de los alumnos/as y su incorporación a la práctica deportiva de manera federada.

No se han encontrado diferencias significativas en los valores de autodeterminación en función del deporte practicado (fútbol, baloncesto, piragüismo, tenis y artes marciales); aunque si cabe destacar que son los jugadores de baloncesto los que presentan unas puntuaciones mayores en motivación intrínseca e identificada. Esto viene a sugerir que su práctica les provoca en mayor medida disfrute, diversión o lo consideran que es importante para su vida (salud, relaciones sociales) mostrando más interés y emociones positivas en su práctica. En el polo contrario, es decir, con un mayor nivel de desmotivación, se encuentran los futbolistas y piragüistas que presentan unas puntuaciones más altas en regulación externa (realizan ese deporte por presión de padres, compañeros/as, entrenadores, etc.) y desmotivación (empiezan a cuestionarse seguir con su práctica). Esto es muy peligroso porque puede provocar aburrimiento (Ntoumanis, 2001), infelicidad (Standage, Duda \& Ntoumanis, 2005) y abandono deportivo (Sarrazin, et al., 2002).

En cuanto a las principales limitaciones de este estudio, el hecho de que sólo se escogieran centros en los que se hubieran llevado a cabo unidades didácticas de piragüismo ha provocado que la población sea pequeña y sesgada. Esto podría explicar que aunque se encuentren ciertas tendencias en función de la práctica físico-deportiva practicada, no haya diferencias significativas entre deportes.

\section{Conclusión}

Los chicos adolescentes practican más deporte que las chicas, especialmente cuando se trata de deporte federado. Los hombres mostraron una mayor motivación intrínseca que las mujeres. Se ha observado que los alumnos que practican deporte federado, indistintamente de la modalidad deportiva que hagan, tienen mayor motivación intrínseca e 
identificada, lo cual favoreceré su continuidad en la práctica. Además, se ha encontrado quelos deportes más practicados son el fútbol, el piragüismo y el baloncesto; por lo que se podría afirmar que la implantación de unidades didácticas en el ámbito escolar relacionadas con un determinado deporte pueden promover la continuidad de los niños en esta modalidad deportiva. Por tanto, se debería incluir dentro del ámbito escolar una mayor oferta deportiva, presentando ésta de manera atrayente, buscando que el niño comience a realizar un ejercicio físico de forma continuada; y a ser posible de forma federada, no buscando únicamente el rendimiento sino cumplir las motivaciones que llevan a los niños a practicar deporte.

\section{Referencias}

American College of Sports Medicine (2000). ACSM's quidelines for exercise testing and prescription ( $6^{\mathrm{a}}$ ed.). Filadelfia, PA: Lippincott Williams y Wilkins.

Armenta, F., Prieto, I., \& Morilla, M. (2003). Motivos que llevan a los jóvenes a la práctica deportiva y causas del abandono prematuro. En Actas del I Congreso Mundial de Ciencias de la Actividad Física y del Deporte (1-5). Granada.

Balaguer, I. (1994). Entrenamiento psicológico del deporte. Valencia: Albatros.

Balaguer, I., Castillo, I., \& Duda, J. (2008). Apoyo a la autonomía, satisfacción de las necesidades, motivación y bienestar en deportistas de competición: un análisis de la teoría de la autodeterminación. Revista de Psicología del Deporte, 17(1), 123-139.

Balaguer, I., Pastor, Y., \& Moreno, Y. (1999). Algunas características de los estilos de vida de los adolescentes de la Comunidad Valenciana. Revista Valenciana d'Estudis Autonómics, 26, 33-56.

Biddle, S., \& Goudas, M. (1996). Analysis of children's physical activity and its association with adult encouragement and social cognitive variables. Journal of School Health, 66(2), 75-78.

Brustad, R. (1996). Attraction to Physical Activity in Urban Schoolchildren: Parental Socialization and Gender Influences. Research Quarterly for Exercise and Sport, 67(3), 316-323.

Cantallops, J., Ponseti, F.J., Vidal, J., Antoni, P., \& Palou, P. (2012). Adolescencia, sedentarismo y sobrepeso: análisis en función de variables sociopersonales de los padres y del tipo de deporte practicado por los hijos. Retos. Nuevas tendencias en Educación Física, Deporte y Recreación, 21, 5-8.

Castillo, I., \& Balaguer, I. (1998). Patrones de actividades físicas en niños y adolescentes. Apunts. Educación Física y Deportes, 54, $22-29$.

Castillo, I., \& Balaguer, I. (2001). Dimensiones de los motivos de práctica deportiva de los adolescentes valencianos escolarizados. Apunts, Educación Física y Deportes, 63, 22-29.

Castillo, I., Balaguer, I., \& Duda, J.L. (2000). Las orientaciones de meta y los motivos de práctica deportiva en los jóvenes deportistas valencianos escolarizados. Revista de Psicología del Deporte, 9(1-2), 37-50.

Castillo, I., Balaguer I., \& Tomás, I. (1997). Predictores de la práctica de actividades físicas en niños y adolescentes. Anales de psicología, 13(2), 189-200.

Fox, K. (2000). The effects of exercice on selfperceptions and self-esteem. En S. Biddle, K. Fox \& S. Boutcher (Eds.), Physical Activity and Psychological Well-Being (pp. 88-118). Londres: Routhledge.

García-Ferrando, M. (2006a). Posmodernidad y deporte: Entre la individualización y la masificación. Encuesta sobre hábitos deportivos de los españoles 2005. Madrid: CSD y CIS.

García-Ferrando, M. (2006b). Veinticinco años de análisis del comportamiento deportivo de la población española (1980-2005). Revista Internacional de Sociología (RIS), 64(44), 15-38.

Greendorfer, S.L., \& Lewko, J. (1978). Role of Family Members in Sport Socialization of Children. Research Quarterly for Exercise and Sport, 49(2), 146-152.

Hellín, P., Moreno, J., \& Rodríguez, P. (2004). Motivos de práctica físico-deportiva en la Región de Murcia. Cuadernos de Psicología del Deporte, 4(1-2), 101116.

Hernández, L.A., Ferrando, J., Quílez, J., Aragonés, M., \& Terreros, J. (2010) Análisis de la actividad física en escolares de medio urbano. (pp. 60-93). Madrid: Subdirección general de deporte y Salud. Consejo Superior de Deportes.

Macías, V., \& Moya, M. (2002) Género y deporte. La influencia de variables psicosociales sobre la práctica deportiva de jóvenes de ambos sexos. Revista de psicología social aplicada, 17(2), 129-148.

Markland, D., \& Tobin, V. (2004). A modification to Behavioural Regulation in Exercise Questionnaire to include an assement of amotivation. Journal of Sport and Exercise Psychology, 26(2), 191-196.

Martínez-Gómez, D., Joey, C., Gómez, S., Veses, A., Marcos, A., \& Veiga, O. (2010). Sedentarismo, adiposidad y factores de riesgo cardiovascular en adolescentes. Estudio AFINOS. Revista Española de Cardiología, 63(3), 277-285.

McPherson, B., Curtis, J., \& Loy, J. (1989). The social significance of sport. An introduction to the sociology of sport. Champaign, IL: Human Kinetics.

Moreno, J. A., Cano, F., González-Cutre, D., Cervelló, E., \& Ruiz, L. (2009). Flow disposicional en salvamento deportivo: una aproximación desde la teoría de la autodeterminación. Revista de Psicología del Deporte, 18(1), 23-35.

Moreno, J.A., Cervelló, E., \& González-Cutre, D. (2007). Young athletes motivational profiles. Journal of Sports Science and Medicine, 6, 172-179.

Moreno, J.A., Cervelló, E., \& Martínez, A. (2007). Measuring selfdetermination motivation in a physical fitness setting; validation of the Behavioral Regulation in Exercise Questionnaire-2 (BREQ-2) in Spanish sample. The Journal of Sports Medicine and Physical Fitness, 47(3), 366-374.

Navarro, M., Ojeda, R., Navarro, M., López, E., Brito, E., \& Ruiz, J.A. (2012). Compromiso de los adolescentes de Canarias con un estilo de vida físicamente activo y saludable. Retos. Nuevas tendencias en Educación Física, Deporte y Recreación, 21, 30-33.

Nicholls, J.G (1989). The competitive ethos and democratic education. Cambridge, MA: Harvard University Press.

Ntoumanis, N. (2001). A self-determination approach to the understanding of motivation in physical education. British Journal of Educational Psychology, 71(2), 225-242.

Nuviala, A., Ruiz, F., \& García, E. (2003). Tiempo libre, ocio y actividad física en los adolescentes. La influencia de los padres. Retos. Nuevas tendencias en Educación Física, Deporte y Recreación, 6, 13-20.

Palou, P., Ponseti, X., Gili, M., Borras, P., \& Vidal., J. (2005). Motivos para el inicio, mantenimiento y abandono de la práctica deportiva de los preadolescentes de la isla de Mallorca. Apunts. Educación física y Deportes, 81, 5-11.

Pallarés, J. (1998). Los agentes psicosociales como moduladores de la motivación en deportistas jóvenes orientados al rendimiento: un modelo causal. Revista de Psicología del Deporte, 7(2), 275-281.

Parcel, G, Simons-Mortons, B., O’Hara, N., Baranowski, T., Kolbe, L., \& Bee, D. (1987). School promotion of healthful diet and exercise behavior: An integration of organizational change and social learning theory interventions. Journal of School Health, 57(4), 150-156.

Pavón, A., \& Moreno, J. A. (2008). Actitud de los universitarios ante la práctica física-deportiva: diferencias por género. Revista de Psicología del Deporte, 17(1), 7-23.

Piéron, M. (2002). Estudi sobre els hàbits esportius dels escolars d'Andorra. Govern d'Andorra, Ministeri d'Educació Joventut i Esports.

Raudsepp, L., \& Viira, R. (2000). Sociocultural Correlates of Physical Activity in Adolescents. Pediatric Exercise Science, 12(1), 51-60.

Roberts, GC. (1992). Motivation in sport an exercise: Conceptual constraints and conceptual convergence. In G.C. Roberts (Ed.), Motivation in sport and exercise (pp. 3-30). Champaign, IL: Human Kinetics.

Romero, S. Garrido, M., \& Zagalaz, M. (2009). The parents behavior in the sports. Retos. Nuevas tendencias en Educación Física, Deporte y Recreación, 15, 2934.

Ryan, R.M., \& Deci, E.L. (2000). Self-determination theory and the facilitation on intrinsic motivation, social development, and well-being. American Psychologist, 55(1), 68-78.

Sallis, J., Prochaska, J., \& Taylor, W. (2000). A review of correlates of physical activity of children and adolescent. Medicine and Science in Sport and Exercise, 32(5), 963-975.

Sallis, J., Simons-Morton, B., Stone, E., Corbin, C., Epstein, L.H.,... \& Taylor,W. (1992). Determinants of physical activity and interventions in youth. Medicine and Science in Sports and Exercise, 24(6 Sppl), S248-S257.

Sansone, C., \& Harackiewicz, J. (2000). Controversias and new directions-is it déjà vu all over again? En C. Sansone, \& J. Harackiewicz (Eds.), Intrinsic and extrinsic motivation: The search for optimal motivation and performance (pp.443-453). San Diego, CA: Academic Press.

Sarrazin, P., Vallerand, R., Guillet, E., Pelletier, L., \& Cury, F. (2002). Motivation and dropout in female handballers: A 21-month prospective study. European Journal of Social Psychology, 31, 395-418.

Shoopshire, J., \& Carroll, B. (1997). Family variables and children's physical activity: Influence of parental exercise and socio-economic status. Sport, Education and Society, 2(1), 95-116.

Sonstroem, R., \& Potts, S. (1996). Life adjustment correlates of physical selfconcepts. Medicine and Science in Sports and Exercice. 28(5), 619-625.

Standage, M., Duda, J., \& Ntoumanis, N. (2003). A model of contextual motivation in physical education: Using constructs from self-determination and achievement goal theories to predict physical activity intentions. Journal of educational psychology, 95(1), 97-110.

Trigo, E. (1990). La actividad deportiva de los jóvenes estudiantes de enseñanzas medias de Galicia durante su tiempo libre. Revista de investigación y documentación sobre las Ciencias de la Educación Física y del Deporte, 15(16), 70-81.

Van Der Horst, K., Paw, M., Twisk, J., \& Van Mechelen, W. (2007). A Brief Review on Correlates of Physical Activity and Sedentariness in Youth. Medicine $y$ Science in Sports y Exercise, 39(8), 1241-1250.

Varo, J., Martínez, J., \& Martínez-González, M. (2003). Beneficios de la actividad física y riesgos del sedentarismo. Medicina Clínica, 121(17), 665-672.

Villamarin, F., Mauri, C., \& Sanz, A. (1998). Competencia percibida y motivación durante la iniciación en la práctica del tenis. Revista de Psicología del Deporte, 13, 41-56.

Vlachopoulos, S., Karageorghis, C., \& Terry, P. (2000). Motivation profiles in sport: A self-determination theory perspective. Research Quarterly for Exercise and Sport, 71(4), 387-397.

Wold, B. (1995). Health behavior in school children: A WHO cross-national survey. Resorce Package of Questions 1993-94, Norway: University of Bergen.

Wuerth, S., Lee, M., \& Alfermann, D. (2004). Parental involvement and athletes in youth sport. Psychology of sport and Exercise, 5(1), 21-33.

Zakarian, J., Hovell, M., Hofstetter, C., Sallis, J. \& Keating, K. (1994). Correlates of vigorous exercise in a predominantly low SES and minority high school population. Preventive Medicine, 23(3), 314-321. 